\title{
Long-period comet impact risk mitigation with Earth-based laser arrays
}

\section{Qicheng Zhang, Philip M. Lubin, Gary B. Hughes}

Qicheng Zhang, Philip M. Lubin, Gary B. Hughes, "Long-period comet impact risk mitigation with Earth-based laser arrays," Proc. SPIE 10401, Astronomical Optics: Design, Manufacture, and Test of Space and Ground Systems, 1040104 (8 September 2017); doi: 10.1117/12.2274726

EDent: SPIE Optical Engineering + Applications, 2017, San Diego, California, United States 


\title{
Long-period comet impact risk mitigation with Earth-based laser arrays
}

\author{
Qicheng Zhang*a,b, Philip M. Lubin ${ }^{\mathrm{a}}$, Gary B. Hughes ${ }^{\mathrm{c}}$ \\ ${ }^{a}$ Dept. of Physics, Univ. of California, Santa Barbara, CA, USA \\ ${ }^{\mathrm{b}}$ Division of Geological and Planetary Sciences, California Institute of Technology, Pasadena, \\ CA, USA

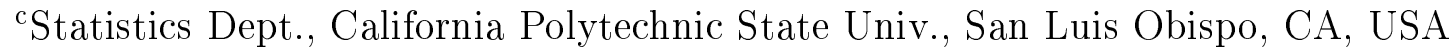

\begin{abstract}
Long-period comets (LPCs) frequently transit the inner solar system, and like near-Earth asteroids (NEAs), pose a continued risk of impact with Earth. Unlike NEAs, LPCs follow nearly parabolic trajectories and approach from the distant outer solar system where they cannot be observed. An LPC on an Earth-impact trajectory is unlikely to be discovered more than a few years in advance of its arrival, even with significant advancements in sky survey detection capabilities, likely leaving insufficient time to develop and deliver an interception mission to deflect the comet. However, recent proposals have called for the development of one or more large $\sim 1 \mathrm{~km}$ laser arrays placed on or near Earth primarily as a means for photon propulsion of low-mass spacecraft at delta-v above what would be feasible by traditional chemical or ion propulsion methods. Such a laser array can also be directed to target and heat a threatening comet, sublimating its ices and activating jets of dust and vapor which alter the comet's trajectory in a manner similar to rocket propulsion. Simulations of directed energy comet deflection were previously developed from astrometric models of nongravitational orbital perturbations from solar heating, an analogous process that has been observed in numerous comets. These simulations are used together with the distribution of known LPC trajectories to evaluate the effect of an operational Earth-based laser array on the LPC impact risk.
\end{abstract}

Keywords: comets, high-powered lasers, phased-array optics, planetary defense

\section{INTRODUCTION}

Comets are small bodies with a significant volatile content, particularly of water ice, that sublimates at points in their orbit sufficiently close to the Sun-typically anywhere in or near the inner solar system. They may be divided into three categories for which the following definitions are adopted in this manuscript:

1. Jupiter-family comets (JFCs) have orbital periods under $20 \mathrm{yr}$. Most of these comets follow orbits with fairly low inclinations below $\sim 30^{\circ}$.

2. Halley-type comets (HTCs) have orbital periods between $20 \mathrm{yr}$ and $200 \mathrm{yr}$.

3. Long-period comets (LPCs) have orbital periods above $200 \mathrm{yr}$ or follow gravitationally unbound trajectories which have no defined orbital periods.

Like near-Earth asteroids (NEAs), many comets follow trajectories that intersect that of Earth, and thus similarly pose a continuing impact threat. This manuscript focuses on the impact threat posed by LPCs. Unlike NEAs, which tend to follow relatively low eccentricity orbits, LPCs follow nearly parabolic trajectories. Consequently, LPCs rarely experience significant solar heating in the inner solar system and thus retain a large fraction of their volatile ices. These properties present a number of important consequences for impact mitigation techniques, should such action prove necessary:

\footnotetext{
* corresponding author - email: qicheng@cometary.org
} 
- Rendezvousing with an LPC, where a spacecraft transfers into the orbit of the comet, is presently infeasible due to the high delta-v required. Therefore, deflection strategies using a gravity tractor ${ }^{1}$ or an ion beam shepherd,$^{2}$ both of which involve a spacecraft in close proximity to the target over an extended duration, are not possible.

- Objects cannot be intercepted by a kinetic impactor spacecraft until they are sufficiently close to the ecliptic plane due, again, to delta-v limitations. ${ }^{3}$ LPCs that approach from a direction far above or below the ecliptic may not be in range until shortly before impact, greatly limiting the effectiveness of the kinetic impactor.

- Due to their approach from the distant outer solar system, LPCs cannot be observed and therefore rarely discovered more than a few years before perihelion, even with significant advancements in sky survey detection capabilities. ${ }^{4}$ Therefore, any response to a threatening LPC must be rapid and fully prepared prior to threat identification to be effective.

- Sublimation of ices on any comet drives jets of gas and dust which can significant alter and thus produce large uncertainties in its future trajectory and therefore any threat of impact. This effect has been observed to be particularly strong in LPCs, likely due to their high volatile content. ${ }^{5}$

The directed energy approach to comet deflection is motivated by the last point and involves purposefully shifting the comet's trajectory by artificially heating the comet with a directed energy source, such as a laser array. Use of phased-array optics can reduce divergence of the laser beam to the diffraction limit and allow the laser to operate from a distance, potentially even from Earth.

Recent proposals have called for the development of one or more $1 \mathrm{~km} / 100 \mathrm{GW}$ laser arrays placed on or near Earth primarily as a means for photon propulsion of low-mass spacecraft at delta-v above what would be feasible by traditional chemical or ion propulsion methods. ${ }^{6}$ Deflection using an Earth-based laser removes the need for a rendezvous or intercept spacecraft and permits immediate action as soon as an impact threat is confirmed.

Directed energy comet deflection simulations were previously developed from the standard astrometric model of nongravitational orbital perturbations from solar heating ${ }^{7}$ by treating laser heating as being equivalent to solar heating. ${ }^{8}$ These simulations were run for a canonical comet based on C/2013 A1 (Siding Siding), an LPC which made a close approach to Mars in 2014, as well as several variants of this comet. These same simulations are used in the following sections to analyze the total LPC impact risk given the presence of an operational Earth-based laser array.

\section{SIMULATIONS}

In order to evaluate LPC impact risk, a set of orbital parameters must be adopted to parametrize all LPC impactors. Consider, at first, the 6 classical orbital elements: perihelion distance $q$, eccentricity $e$, inclination $i$, argument of perihelion $\omega$, longitude of the ascending node $\Omega$, and time of perihelion $T_{\mathrm{p}}$. A first simplification is to take $e=1.0$ for LPCs. In addition, for an impact to occur, the comet's position is constrained to match that of Earth's at impact time $T$ which replaces 3 additional degrees of freedom. Furthermore, the impact problem is approximately invariant in $T$, given the low eccentricity of Earth's orbit and neglecting the gravity of the Moon and planets, leaving 2 independent degrees of freedom to describe the impactor.

A native choice might be to select $q$ and $i$, a pair that, while physically intuitive, is insufficient to fully describe the orbit of an LPC impactor. Up to 4 distinct impact trajectories exist for a given $q$ and $i$ : a pair with impact is at the ascending node and another pair with impact at the descending node, with each pair containing a pre-perihelion impact trajectory and a post-perihelion impact trajectory.

Instead, consider the comet's final direction of approach to Earth in the solar rest frame-the direction opposing the comet's heliocentric velocity at impact. This direction can described by $\alpha$ : ecliptic longitude, and $\beta$ : ecliptic latitude, with an appropriate $T$ near the September equinox such that $\alpha=0$ is the anti-solar direction. Every Earth impactor must approach the Earth from a direction, and every unique approach direction maps to a unique approach trajectory, indicating that the approach direction alone is fully descriptive of an LPC impactor given the approximations stated above. 


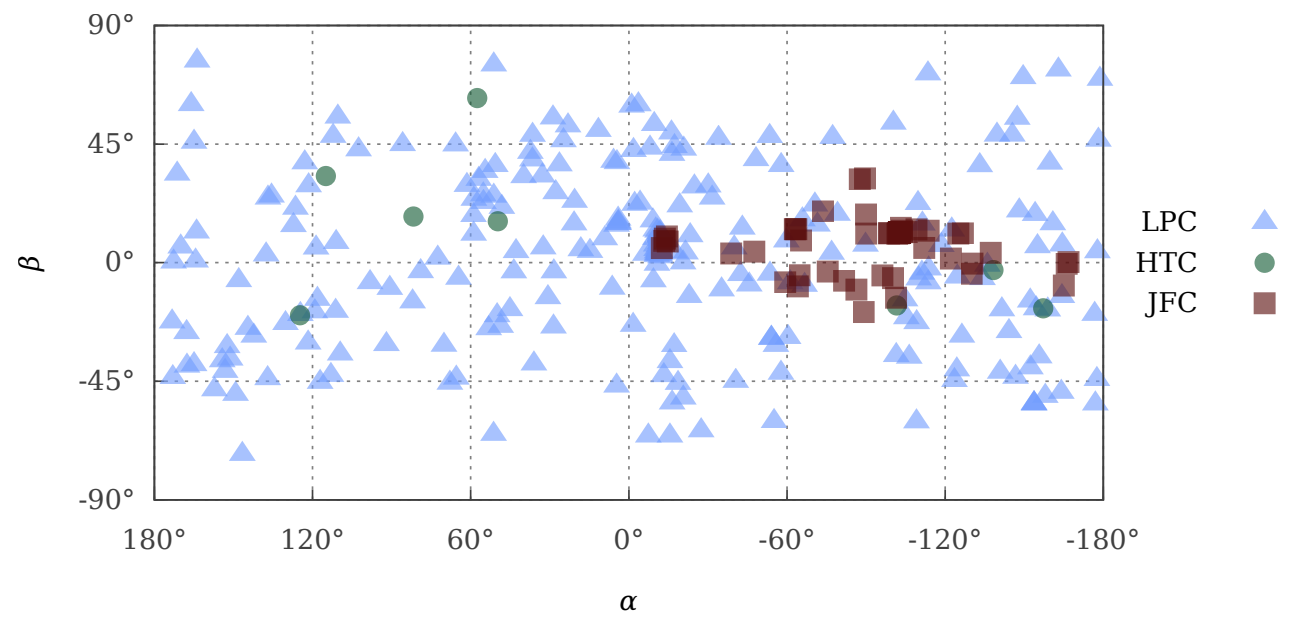

Figure 1. Direction of approach to Earth, in the solar rest frame, of all comets with an Earth minimum orbit intersection distance (MOID) < 0.1 au in the JPL Small-Body Database as of July 7, 2017. Approach direction, computed for each comet's position at MOID, is given in ecliptic coordinates, where $\beta$ is ecliptic latitude and $\alpha$ is ecliptic longitude defined such that $\alpha=0$ indicates the anti-solar direction.

Fig. 1 compares the direction of approach in the solar rest frame of all comets with an Earth minimum orbit intersection distance (MOID) $<0.1$ au in the JPL Small-Body Database as of July 7, 2017, including 278 LPCs. Unlike JFCs which are confined to a narrow range of low-inclination $(\beta$ near 0$)$ prograde $(\alpha<0)$ orbits, LPCs (and HTCs) do not appear to be confined in approach direction. An LPC impactor orbit sample is obtained from these 278 LPCs by fitting trajectories with $e=1.0$ through Earth at time $T$ with the provided $\alpha$ and $\beta$.

\subsection{Orbital Model}

Orbital propagation uses the comet deflection model described in Zhang et al. (2016). ${ }^{8}$ This model is based on the astrometric model of Marsden et al. $(1973)^{7}$ for a spherically-symmetric, non-rotating comet which gives the radial component of acceleration from solar heating as

$$
\ddot{r}=A \times \alpha_{\mathrm{NG}}\left(\frac{r}{r_{0}}\right)^{-m}\left(1+\left(\frac{r}{r_{0}}\right)^{n}\right)^{-k}
$$

at a distance $r$ from the Sun, with $r_{0}=2.808 \mathrm{au}, \alpha_{\mathrm{NG}}=0.111262, m=2.15, n=5.093$, and $k=4.6142$, where the constant of proportionality $A$ is the nongravitational parameter that differs between comets, with $A \sim 10^{-7} \mathrm{au} \cdot \mathrm{d}^{-2}$ for LPCs, but can vary by over an order of magnitude. ${ }^{5}$

The full orbital model combines the heating by the Sun and by the laser beam, and assumes an equivalent response to heating from both sources. The laser beam, of total power $P_{\text {las }}$, is approximated as a uniform beam diverging at a half angle $\delta \theta=\lambda_{\text {las }} / L_{\text {las }}$, where $\lambda_{\text {las }}$ is the wavelength of the beam, and $L_{\text {las }}$ is the characteristic width of the laser array. Due to energy and heat dissipation requirements, the laser arrays may only for short periods at a time. The maximum fraction of a day a laser may operate is denoted by $\tau$.

Terrestrial laser arrays are assumed to be equipped with adaptive optics sufficiently powerful such that they produce a diffraction-limited beam of quality comparable to that produced by an orbital laser array. Terrestrial lasers, however, are constrained to pointing within a field of view of diameter $\Theta_{\text {fov }}$ centered on their zenith and may only target the comet for the fraction of each day the comet is within this field of view which is a function of the laser's latitude. In addition, due to local weather, terrestrial lasers are further suppressed by a factor $\kappa$, the fraction of time conditions are conducive to the use of the laser.

The comets are orbitally propagated under the model described above either to impact, or to the point of closest approach to Earth if no impact occurs. Deflection distance $\Delta_{\text {def }}$ is defined to be the shift in the target 


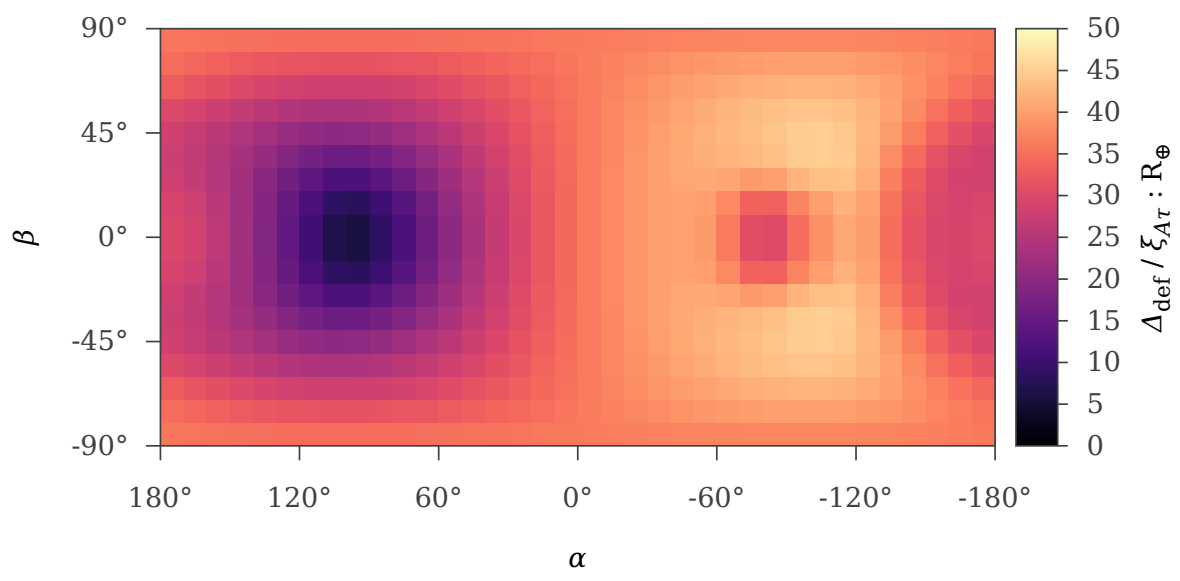

Figure 2. Deflection distance dependence on approach direction for a $D_{\text {com }}=5 \mathrm{~km}$ LPC deflected by a $L_{\text {las }}=1 \mathrm{~km}$ orbital laser array producing a $\lambda_{\text {las }}=1 \mu \mathrm{m}$ beam at $P_{\text {las }}=100 \mathrm{GW}$ over $2 \mathrm{yr}$.

plane crossing point of the comet. In this case of an impact, the target plane crossing point is defined to be that of the linear extension to the physical trajectory.

Previous simulation results ${ }^{8}$ found the scaling relations $\Delta_{\text {def }} \propto A$ and $\Delta_{\text {def }} \propto \tau$. All deflection results are only evaluated for $\Delta_{\text {def }}$ in the single reference case $A=A_{0} \equiv 10^{-7} \mathrm{au} \cdot \mathrm{d}^{-2}$ and $\tau=\tau_{0} \equiv 10 \mathrm{~min} \cdot \mathrm{d}^{-1}$ which gives a normalized deflection distance $\Delta_{\text {def }} / \xi_{A \tau}$, where $\xi_{A \tau} \equiv\left(A / A_{0}\right) \times\left(\tau / \tau_{0}\right)$, that can be scaled for $\Delta_{\text {def }}$ for any $A$ and $\tau$.

\section{RESULTS}

For brevity, this analysis is limited to consideration of the class of laser arrays recently proposed for propelling near-relativistic wafer spacecraft as a means interstellar exploration. ${ }^{6}$ Specifically, this analysis considers an $L_{\text {las }}=1 \mathrm{~km}$ phased laser array producing a beam of $\lambda_{\text {las }}=1 \mu \mathrm{m}$ at $P_{\text {las }}=100 \mathrm{GW}$.

While $A$ can be determined astrometrically for many LPCs, ${ }^{5}$ reliable estimates of comet diameter $D_{\text {com }}$ requires direct observation of the nucleus, and so are only available in a select few instances. For example, LPCs with radar measurements include C/1983 H1 (IRAS-Araki-Alcock) at $D_{\text {com }}=8.8 \mathrm{~km}, \mathrm{C} / 1983 \mathrm{~J} 1$ (SuganoSaigusa-Fujikawa) at $D_{\text {com }}=0.74 \mathrm{~km}$ and C/1996 B2 (Hyakutake) at $D_{\text {com }}=2.5 \mathrm{~km} .{ }^{9}$ Unlike $A$, there is no simple scaling relation relating $\Delta_{\text {def }}$ with $D_{\text {com }}$, so $\Delta_{\text {def }}$ must be separately evaluated for each $D_{\text {com }}$ to be considered.

Fig. 2 shows the deflection distance dependence on approach direction for a $D_{\text {com }}=5 \mathrm{~km}$ LPC deflected by the aforementioned laser array over the $2 \mathrm{yr}$ before its Earth encounter in the absence of weather interference and pointing restrictions (i.e., an orbital laser array). Overall, deflection is significantly more effective against prograde impactors than retrograde impactors, with the variation between extrema spanning an order of magnitude. Deflection also appears to be more effective with a moderately inclined approach trajectory than with low inclination, a contrast to any spacecraft interception method which is most effective with impactors approaching from near the plane of the ecliptic.

Directional bias is further amplified for terrestrial laser arrays by restrictions in pointing direction. Fig. 3 shows the deflection effectiveness of terrestrial laser arrays of the same parameters as the previous orbital laser array placed at latitude $20^{\circ} \mathrm{N}$ and $25^{\circ} \mathrm{S}$ with favorable weather for $\kappa=0.75$ - comparable to Mauna Kea and Paranal Observatory respectively. ${ }^{10,11}$ These results are consistent with the overall pattern that a laser array in the Northern Hemisphere favors deflection of comets approaching from north of the ecliptic, while a laser in the Southern Hemisphere is most effective against comets approaching from south of the ecliptic. Approach 

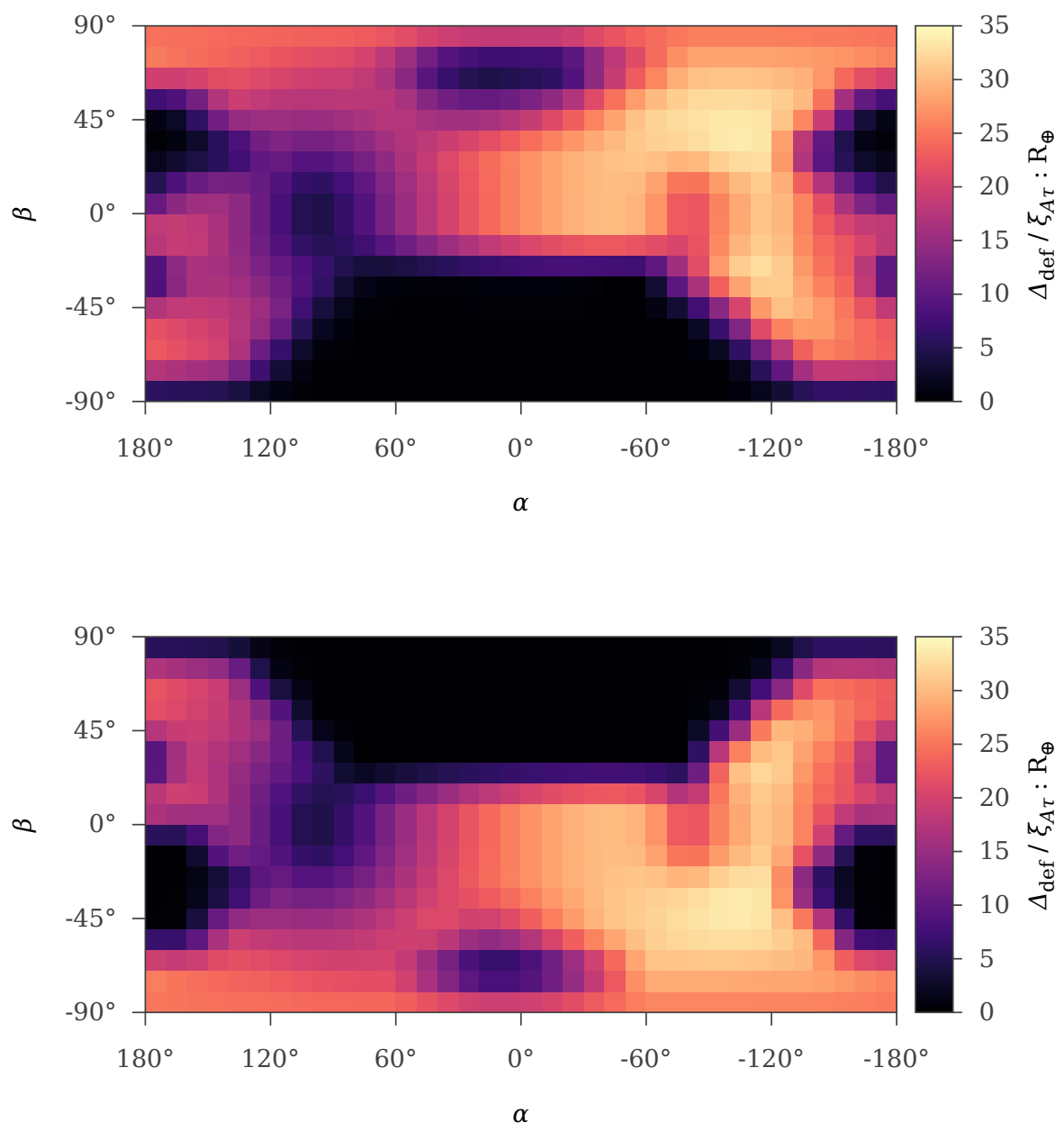

Figure 3. Direction bias in deflection effectiveness for a terrestrial laser array placed at $20^{\circ} \mathrm{N}$ (above) and $25^{\circ} \mathrm{S}$ (below), with $\kappa=0.75$ for both cases. Laser and comet parameters are otherwise identical to those given for Fig. 2.

directions in the opposite hemisphere fall into a large "blind spot" corresponding to comets that never pass sufficiently closely to the local zenith for the laser to ever operate.

An exception to this pattern occurs for comets approaching from $\alpha \sim \pm 180^{\circ}$, in the general direction of the Sun. These comets encounter Earth after perihelion on the outbound leg of their parabolic trajectory, traveling in nearly the opposite direction as on the inbound leg. Thus, a comet approaching Earth from south of the Sun originally approached the inner solar system from north of the ecliptic, favoring deflection by a Northern Hemisphere laser, and vice versa.

To evaluate overall effectiveness at mitigating the LPC impact risk, consider the sample of 278 LPC orbits plotted in Fig. 1, and assume a diameter $D_{\text {com }}=5 \mathrm{~km}$. Fig. 4 compares the distribution of $\Delta_{\text {def }} / \xi_{A \tau}$ for LPCs in this sample deflected by the previously described orbital laser array, and the terrestrial laser arrays at $20^{\circ} \mathrm{N}$ and $25^{\circ} \mathrm{S}$. While a majority of the LPCs in the sample are deflected by the orbital laser by $\Delta_{\text {def }} / \xi_{A \tau} \sim 25-$ $40 \mathrm{R}_{\oplus}$ - close to the maximum value-LPCs deflected by the terrestrial lasers follow a much flatter distribution from just above zero deflection (corresponding to an approach direction in a blind spot) to a maximum of $\Delta_{\text {def }} / \xi_{A \tau} \sim 30 \mathrm{R}_{\oplus}$, a consequence of the stronger directional dependence of terrestrial laser arrays.

Note that the true LPC distribution has non-zero spread in $D_{\text {com }}$ which translates into a wider spread in 


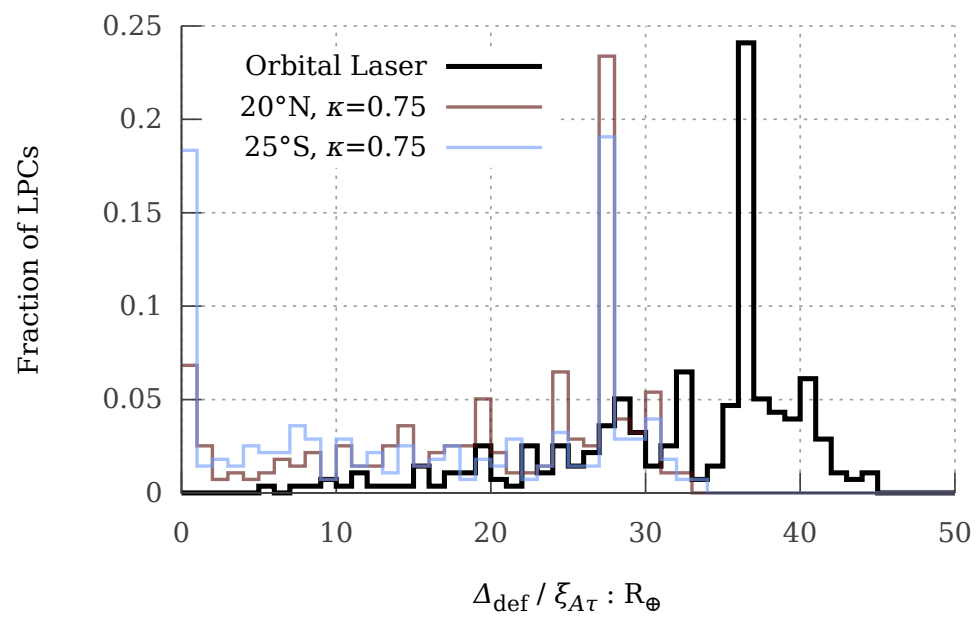

Figure 4 . Histogram of normalized deflection distance $\Delta_{\text {def }} / \xi_{A \tau}$ of the same $D_{\text {com }}=5 \mathrm{~km}$ LPC over 2 yr with approach directions given by the LPC sample plotted in Fig. 1 deflected by the same $L_{\text {las }}=1 \mathrm{~km}, P_{\text {las }}=100 \mathrm{GW}$ laser arrays considered in Fig. 2 and Fig. 3 in orbit, and at $20^{\circ} \mathrm{N}$ and $25^{\circ} \mathrm{S}$. Bin size is $1 \mathrm{R}_{\oplus}$.

the actual distribution of $\Delta_{\text {def }} / \xi_{A \tau}$. These results, however, provide a lower bound to $\Delta_{\text {def }} / \xi_{A \tau}$ for LPCs of $D_{\text {com }}<5 \mathrm{~km}$.

\section{CONCLUSIONS}

No LPC impact events have ever occurred in recorded history, and it is likely none will occur anytime in the foreseeable future, so it is likely no defense system will be necessary in the upcoming decades or even centuries. Nonetheless, LPC impacts present a serious hazard to the well-being of life on Earth due to the severity of effects from even a single event which are likely to be of global consequence given the typical sizes and speeds of these objects. ${ }^{12}$ Moreover, the majority of large asteroids have already been discovered and are being tracked so that any impact will provide centuries of warning rather than the few years provided by LPCs of any size.

The results presented in this manuscript show that adequate defense from LPC impacts is possible without a dedicated planetary defense system. A laser array of a scale being proposed near-relativistic space probe propulsion can be effectively adapted to target LPC impactors within the laser's field of view, and safely deflect them over $2 \mathrm{yr}$ with just a few minutes of activity each day. While an individual terrestrial laser array has a limited range of pointing directions, a network of such laser arrays covering both hemispheres can collectively target LPCs approaching Earth from any direction, effectively mitigating the long-term LPC impact threat.

\section{ACKNOWLEDGMENTS}

We gratefully acknowledge funding from the NASA California Space Grant NNX10AT93H and from NASA NIAC NNX15AL91G and NNX16AL32G as well as a generous gift from the Emmett and Gladys W. Fund in support of this research. GNU Parallel was used in generating the presented simulation results. ${ }^{13}$

\section{REFERENCES}

1. E. T. Lu and S. G. Love, "Gravitational tractor for towing asteroids," Natur 438(7065), p. 177, 2005.

2. C. Bombardelli and J. Peláez, "Ion beam shepherd for asteroid deflection," JGCD 34(4), pp. 1270-1272, 2011.

3. C. R. McInnes, "Deflection of near-earth asteroids by kinetic energy impacts from retrograde orbits," PESS 52(7), p. 587, 2004.

4. P. J. Francis, "The demographics of long-period comets," ApJ 635(2), p. 1348, 2005. 
5. M. Królikowska, "Long-period comets with non-gravitational effects," A\&GA 427(3), pp. 1117-1126, 2004.

6. P. Lubin, "A roadmap to interstellar flight," JBIS 69, pp. 40-72, 2016.

7. B. G. Marsden, Z. Sekanina, and D. Yeomans, "Comets and nongravitational forces. v," AJ 78, p. 211, 1973.

8. Q. Zhang, P. M. Lubin, and G. B. Hughes, "Simulations of directed energy comet deflection," in Proc. SPIE, G. B. Hughes, ed., Planetary Defense and Space Environment Applications 9981, p. 998108, sep 2016.

9. J. Harmon, D. Campbell, S. Ostro, and M. Nolan, "Radar observations of comets," P\&SSS 47(12), pp. 1409$1422,1999$.

10. D. Morrison, R. Murphy, D. Cruikshank, W. Sinton, and T. Martin, "Evaluation of mauna kea, hawaii, as an observatory site," PASP 85(505), p. 255, 1973.

11. A. Ardeberg, I. Lundstrom, and H. Lindgren, "La silla and paranal - a comparison of photometric qualities," AEA 230, pp. 518-526, Apr. 1990.

12. D. K. Yeomans and A. B. Chamberlin, "Comparing the earth impact flux from comets and near-earth asteroids," AcAau 90, pp. 3-5, Sept. 2013.

13. O. Tange, "Gnu parallel - the command-line power tool," ;login: The USENIX Magazine 36, pp. 42-47, Feb 2011. 